\title{
Evaluation and pilot implementation of essential interventions for the management of hypertension and prevention of cardiovascular diseases in primary health care in the Republic of Tajikistan
}

\author{
Dylan Collins ${ }^{1 *}$, Laura Inglin², Tiina Laatikainen ${ }^{2,3,4}$, Mekhri Shoismatuloeva ${ }^{5}$, Dilorom Sultonova ${ }^{6}$, Bunafsha Jonova ${ }^{7}$, \\ Katoyon Faromuzova ${ }^{8,9}$, Marifat Abdullaeva', Maisara Otambekova ${ }^{1}$ and Jill L. Farrington ${ }^{5}$
}

\begin{abstract}
Background: The aim of this study was to determine the feasibility of implementing and evaluating essential interventions for the management of hypertension and prevention of cardiovascular disease in primary healthcare in Tajikistan.

Methods: The study protocol was published a priori. A pragmatic, sequential, mixed methods explanatory design was piloted. The quantitative strand is reported here. All primary health care facilities that met inclusion criteria in Shahrinav district were included and computer randomized to either usual care or intervention. The intervention consisted of: adaptation of WHO PEN/HEARTS clinical algorithms for hypertension and diabetes, a two-day training of doctors and nurses, supportive supervision visits, clinical decision support tools, and quality improvement support. Data were collected from paper-based clinical records at baseline and 12 months follow-up. The primary outcome was blood pressure control among patients with hypertension, in addition to several secondary process indicators along the care pathway. Age and sex adjusted logistic regression models were used for intervention and control clinics to determine changes between baseline and follow-up and to assess interactions between allocation group and time. For continuous variables, multivariate linear regression models were used.
\end{abstract}

Results: 19 primary health care centres were included of which ten were randomized to intervention and nine to control. 120 clinicians received training. The records of all registered hypertensive patients were reviewed at baseline and follow-up for a total of 1,085 patient records. Blood pressure control significantly improved in the intervention clinics (OR 3.556, $95 \% \mathrm{Cl} 2.219,5.696)$ but not the control clinics (OR 0.644, $95 \% \mathrm{Cl} 0.370,1.121)(p<$ 0.001 for interaction). Smoking assessment, statin prescribing, triple therapy prescribing, and blood pressure measurement significantly improved in intervention clinics relative to control, whereas cholesterol and glucose testing, and aspirin prescribing did not.

(Continued on next page)

\footnotetext{
*Correspondence: drjc.ubc@gmail.com

'University of British Columbia, Vancouver, Canada

Full list of author information is available at the end of the article
}

(c) The Author(s). 2021 Open Access This article is licensed under a Creative Commons Attribution 4.0 International License, which permits use, sharing, adaptation, distribution and reproduction in any medium or format, as long as you give appropriate credit to the original author(s) and the source, provide a link to the Creative Commons licence, and indicate if changes were made. The images or other third party material in this article are included in the article's Creative Commons licence, unless indicated otherwise in a credit line to the material. If material is not included in the article's Creative Commons licence and your intended use is not permitted by statutory regulation or exceeds the permitted use, you will need to obtain permission directly from the copyright holder. To view a copy of this licence, visit http://creativecommons.org/licenses/by/4.0/ The Creative Commons Public Domain Dedication waiver (http://creativecommons.org/publicdomain/zero/1.0/) applies to the data made available in this article, unless otherwise stated in a credit line to the data. 
(Continued from previous page)

Conclusions: It is feasible to use routine, paper-based, clinical records to evaluate essential CVD interventions in primary health care in Tajikistan. Adapted WHO PEN/HEARTS guidelines in the context of a complex intervention significantly improved blood pressure control after 12 months.

\section{Background}

Non-communicable diseases (NCDs) are the leading cause of death worldwide and are particularly prevalent in Tajikistan. [1] The risk of premature mortality (death before the age of 70) from the four major NCDs (cardiovascular diseases, diabetes, cancer, and chronic respiratory disease) is $25 \%$ with men being disproportionally affected. [2] This is in part due to the high population prevalence of tobacco and alcohol use among men, in addition to a $47 \%$ prevalence of overweight in the adult population. [3] These trends also hold true in Tajikistan.

The detection and management of cardiovascular disease risk in primary health care is an urgent priority given the burden of cardiovascular disease (CVD) and the current suboptimal state of primary health care in Tajikistan. Population health data is scarce in Tajikistan but the best estimates to our knowledge, although not publicly available, are WHO STEPS survey data which estimates that $44.3 \%$ of men and $24.7 \%$ of women have never had their blood pressure measured and the prevalence of high CVD risk among adults aged 40-69 is $13.8 \%$. [3]

Early efforts in Tajikistan to build primary health care capacity and implement essential interventions for CVD in primary health care had mixed success.[4] From 2013 through to 2015 Tajikistan adapted and piloted the WHO Package of Essential NCD Interventions for Primary Healthcare in Low Resource Settings (WHO PEN). [5] WHO PEN provides an integrated approach, through the use of total cardiovascular risk assessment, to the detection and management of cardiovascular disease risk factors. While risk stratifying and targeting those at highest risk is a "best buy" intervention in terms of cost effectiveness, the initial piloting failed to achieve demonstrable health gains. [6] Some of the key shortcomings were that there was not support for primary health care workers following training, high turnover of staff, and no feedback on performance.

With renewed intent and led by the Ministry of Health and Social protection, in 2018 Tajikistan endeavoured to pilot and evaluate the newly developed HEARTS technical package, which aimed to provide a strategic approach to improving cardiovascular health including simplified clinical guidelines. [7] Together with the WHO Regional Office for Europe and the WHO Country Office of Tajikistan, the Ministry of Health and Social Protection convened a national steering group tasked with adapting, piloting, and evaluating the WHO
PEN/HEARTS technical package with a particular focus on systems monitoring, building sustainable and scalable approaches, and integrating with other health systems reforms.

To achieve this, a complex intervention including a two-day training course on the prevention and management of CVD risk, clinical guidelines, paper-based decision support tools, and implementation guidance was developed and piloted in one region of Tajikistan. The aim of this study was to determine the feasibility of implementing and evaluating the complex intervention and its impact on the management of hypertension in primary health care. [3]

\section{Methods}

The study protocol including methods has been previously published. [3] Herein we report the findings from the quantitative strand of a mixed methods study and the abbreviated methods below. Please refer to the published protocol for further methodological details and a broader overview of the study process. [3]

The objectives were to: (1) determine the baseline performance of primary health care facilities; (2) to estimate the change in clinical practice over a 12 month period; and (3) to determine the feasibility of collecting quantitative data required for future studies of effectiveness. An additional objective, to assess the ability to train primary health care clinicians and implement Tajikistanadapted WHO PEN/HEARTS tools, is not reported here and is addressed in the qualitative strand of the study design.

A pragmatic, sequential-mixed methods explanatory design was used. A single district (Shahrinav) was selected based on its nomination by the Ministry of Health, its year-round road access, accessibility from Dushanbe, and lack of exposure to previous interventions. Due to resource constraints and ongoing primary health care reform projects, it was not possible to work in more than one district.

All primary health care centres in Shahrinav were eligible, unless they met one or more exclusion criteria. The exclusion criteria were: [1] city health centre designation (these centres are not nationally representative of primary health care) [2] presence of narrow specialists (i.e. medical specialists other than family medicine) [3] clinics with a family doctor to patient ratio of 1:4000 or more or [4] clinics where information on the number of family doctors and/or family doctor to patient ratio is 
not available.[3] Included clinics were then randomized to either intervention or usual care arms using computer randomization.

While the full description has been previously published, [3] a brief description of the intervention components are as follows:

- One-page clinical protocols for hypertension and diabetes adapted from the WHO HEARTS technical package [7] and WHO PEN [8] in line with national guidelines.

- In-person two-day training integrating adult learning principles and focusing on practical clinical skills covering topics such as measurement and clinical management of CVD risk factors, task sharing, recorded keeping, and patient education material.

- Supportive supervision visits and peer review visits and discussion.

- Paper-based clinical decision support tools including WHO/ISH CVD risk charts, BMI charts, behavioural risk factor counselling.

- Quality improvement and management tools including quarterly monitoring visits using the HEARTS monitoring tool and direct support to improve paper based record keeping systems [7].

The primary outcome was the proportion of hypertensive patients whose blood pressure was controlled, as defined by the number of patients with confirmed hypertension who visited the clinic at least once in the previous 12 months, whose most recent blood pressure reading was controlled $(\mathrm{SBP}<140$ and $\mathrm{DBP}<90)$. A priori secondary outcomes have been previously published and a complete list of indicators are shown in Table 1. [3]

Two cross sectional samples were made, one before and one after implementation; patients from the first sample were not followed longitudinally. Each primary health care centre maintains a register of hypertensive patients and this was used for randomly sampling medical records. At baseline, the sample size was 50 records per clinic and this was adapted to include all patients on the register at follow-up because of low sample sizes at baseline. Inclusion criteria for records were that they were sampled from the hypertension register, that the patient had visited in the last 12 months, and that the patient was at least 18 years or older 12 months prior to the date of data extraction. Data were extracted into a standardized data collection form and entered into excel.

A $10 \%$ point difference in the primary outcome between the intervention and control arms could be detected having 310 to 350 observations per arm at baseline and follow-up using a 0.05 type I error rate and
0.2 type II error rate. A target sample size of 400 per arm was therefore set to ensure ample data.

Age and sex adjusted logistic regression models were used for intervention and control clinics to determine changes between baseline and follow-up and to assess interactions between allocation group and time. Effect estimates were adjusted for age, sex, and clinic to account for clustering at the clinic level. For continuous variables, multivariate linear regression models were used. P-values of $<0.05$ were considered statistically significant. WHO/ISH CVD risk scores were calculated using the whoishRisk package in R. [9]

The study team included local physicians, nurses, academics, WHO national office and European office representatives and worked in close collaboration with the Ministry of Health. A national team of experts adapted all intervention materials (e.g. clinical algorithms) and conducted in person training of health professionals with support from the entire study team. A small team of specially trained national experts used standardized data extraction sheets to record pre-specified routine data that were used to calculate both process and outcome indicators. [3]

\section{Results}

In Shahrinav district, 19 clinics met inclusion criteria and ten were randomized to intervention and nine were randomized to usual care. In total, 15 doctors and 105 nurses were trained. At baseline, 260 and 207 unique patient records were included in the intervention and control group sample, respectively (Table 2). At follow-up, this increased to 374 and 244 unique records, respectively. This translates to a total sample of 1,085 with 467 at baseline and 618 at follow-up. Age distributions were similar between intervention and control at baseline and follow-up. The proportion of newly diagnosed hypertension patients increased significantly in the intervention clinics (from 25 to $47 \% p<0.001$ ) but not the control clinics (from 14 to $20 \% p=0.091$ ); however this increase was not significantly different in the intervention compared to the control ( $p=0.184$ for interaction).

There was no significant difference between intervention and control groups with respect the change in proportion of those who smoke, had diabetes, or a history of CVD (Table 3). There was a statistically significant reduction in systolic and diastolic blood pressure among the intervention group but not the control group $(p<$ $0.001)$. In the intervention group, mean systolic blood pressure was reduced by $8.51 \mathrm{mmHg}(95 \% \mathrm{CI}-10.65$, -6.43) (Table 3). With respect to CVD risk factors among those sampled, too few patients had documented total cholesterol or fasting blood glucose to calculate a reasonable estimate. 
Table 1 Definitions of indicators in Tables 3 and 4

\section{Risk factors}

Current smoker

Diabetes

History of CVD

SBP/DBP

\section{Process indicators}

Smoking status recorded

BP measured regularly

Fasting glucose tested

Total cholesterol tested

WHO/ISH risk score documented

WHO/ISH risk score calculatable

High-risk patients

BP lowering drug prescribed

Statin prescribed for high-risk patients

Aspirin prescribed for high-risk patients

Triple therapy prescribed for highrisk patients

Proportions of smokers. Patients were assumed to be non-smokers if smoking status was not specified.

Proportion of patients with a medical diagnosis ${ }^{a}$ of diabetes mellitus. Patients were assumed to be non-diabetic if diabetes status was not specified.

Proportion of patients with a medical diagnosis ${ }^{\mathrm{b}}$ of cardiovascular disease

Mean based on the most recent systolic/diastolic blood pressure measurement ${ }^{c}$

Proportion of patients with smoking status recorded

Proportion of patients with two documented BP measurements

Proportions of patients with fasting glucose tested

Proportions of patients with total cholesterol tested

Proportions of patients aged 40 years or more with a WHO/ISH CVD risk score documented by health workers based on age, sex, $\mathrm{BP}$, smoking status, diabtes status, and total blood cholesterol

Proportions of patients aged 40 years or more with all risk factors (age, sex, BP, smoking status, diabetes status, and total blood cholesterol) recorded to calculate ${ }^{d}$ the WHO/ISH CVD risk score. Patients were assumed to be non-smoker if smoking status was not specified and to be non-diabetic if diabetes status was not specified. Predictions charts without total cholesterol were applied for those without measurement. The most recent BP and total cholesterol had to be during the last 24 months.

Proportions of patients with a calculated ${ }^{d}$ WHO/ISH $\geq 30 \%$ or/and a history of CVD

Proportions of patients with BP lowering drug prescribed

Proportions of high-risk patients (calculated ${ }^{d}$ WHO/ISH $\geq 30 \%$ or/and history of CVD

Proportions of high-risk patients (calculated ${ }^{d} \mathrm{WHO} / \mathrm{ISH} \geq 30 \%$ or/and history of CVD) prescribed aspirin

Proportions of high-risk patients (calculated ${ }^{\mathrm{d}} \mathrm{WHO} / \mathrm{ISH} \geq 30 \%$ or/and history of CVD) prescribed triple therapy (statin, aspirin, and blood pressure medication)

\section{Outcome indicator}

$\mathrm{BP}$ at normal range

High-risk patients with BP at normal range

Proportion of patients whose most recent $\mathrm{BP}$ measurement ${ }^{c}$ was at normal range $(\mathrm{SBP}<140 \mathrm{mmHg}$ and $\mathrm{DBP}<90 \mathrm{mmHg})$

Proportion of high-risk patients (calculated ${ }^{d} \mathrm{WHO} / \mathrm{ISH} \geq 30 \%$ or/and history of $\mathrm{CVD}$ ) whose most recent BP measurement ${ }^{c}$ was at normal range (SBP $<140 \mathrm{mmHg}$ and $\mathrm{DBP}<90 \mathrm{mmHg}$ )

Low-risk patients with BP at normal Proportion of low-risk patients (calculated ${ }^{d}$ WHO/ISH $<30 \%$ and no history of CVD) whose most recent BP measurement ${ }^{c}$ was at range

CVD cardiovascular disease; SBP systolic blood pressure; DBP diastolic blood pressure; BP blood pressure; WHO/ISH risk score, World Health Organization/ International Society of Hypertension cardiovascular risk score.

${ }^{a}$ Diagnoses written either using ICD-10 codes or as names of diseases to the section of permanent diagnoses in the patient records are taken into account

${ }^{b}$ Diagnoses written either using ICD-10 codes or as names of diseases or events in the patient records are taken into account.

c Measured during the last 12 months.

${ }^{\mathrm{d}}$ Calculated by the authors of this study.

Table 2 Demographic characteristics of patients at baseline and 1-year follow-up data collection

\begin{tabular}{|c|c|c|c|c|}
\hline \multirow[t]{2}{*}{ Characteristics } & \multicolumn{2}{|l|}{ Intervention } & \multicolumn{2}{|l|}{ Control } \\
\hline & Baseline $(N=260)$ & Follow-up $(N=374)$ & Baseline $(N=207)$ & Follow-up $(N=244)$ \\
\hline \multicolumn{5}{|l|}{ Sex } \\
\hline Female, \% (n) & $53.1(138)$ & $63.9(239)$ & $55.6(115)$ & $62.3(152)$ \\
\hline Male, \% (n) & $46.9(122)$ & $36.1(135)$ & $44.4(92)$ & $37.7(92)$ \\
\hline Age, median (IQR) & $62(57-69)$ & $62(57-68)$ & $64(56-71)$ & $63.5(58-70)$ \\
\hline 29-49 years, \% (n) & $6.2(16)$ & $8.0(30)$ & $5.3(11)$ & $4.1(10)$ \\
\hline $50-59$ years, $\%(n)$ & $30.8(80)$ & $30.2(113)$ & $32.9(68)$ & $30.7(75)$ \\
\hline 60-69 years, \% (n) & $40.0(104)$ & $40.9(153)$ & $32.9(68)$ & $37.3(91)$ \\
\hline 70-79 years, \% (n) & $15.0(39)$ & $14.2(53)$ & $21.7(45)$ & $20.9(51)$ \\
\hline $80+$ years, $\%(n)$ & $8.1(21)$ & $6.7(25)$ & $7.2(15)$ & $7.0(17)$ \\
\hline
\end{tabular}

IQR interquartile range 
Table 3 Risk factors for intervention and control clinics at baseline and 1-year follow-up

\begin{tabular}{|c|c|c|c|c|c|}
\hline \multirow[t]{2}{*}{ Risk factors } & \multirow[t]{2}{*}{ Baseline $(N=467)$} & \multirow[t]{2}{*}{ Follow-up $(N=618)$} & \multicolumn{2}{|l|}{ Baseline vs. Follow-up Difference $^{a}$} & \multirow{2}{*}{$\begin{array}{l}\text { Interaction }^{\mathbf{b}} \\
P \text {-value }\end{array}$} \\
\hline & & & OR $(95 \% \mathrm{Cl}) /$ difference in means & $P$-value & \\
\hline \multicolumn{6}{|l|}{ Intervention } \\
\hline Current smoker, \% (n/N) & $5.4(14 / 260)$ & $7.2(27 / 374)$ & $2.059(0.933-4.545)$ & 0.074 & 0.829 \\
\hline Diabetes, \% (n/N) & $15.8(41 / 260)$ & $19.3(72 / 374)$ & $1.354(0.872-2.102)$ & 0.177 & 0.973 \\
\hline Newly diagnosed hypertension, \% (n/N) & $25.4(66 / 260)$ & $47.1(176 / 374)$ & $2.520(1.743-3.643)$ & $<0.001$ & 0.184 \\
\hline History of CVD, \% (n/N) & $20.0(52 / 260)$ & $13.1(49 / 374)$ & $0.653(0.397-1.074)$ & 0.093 & 0.537 \\
\hline $\mathrm{SBP}$, mean $\pm \mathrm{SD}$ & $146.52 \pm 14.25$ & $137.80 \pm 12.07$ & $-8.51(-10.65-6.37)$ & $<0.001$ & $<0.001$ \\
\hline $\mathrm{DBP}$, mean $\pm \mathrm{SD}$ & $91.80 \pm 9.04$ & $86.61 \pm 7.85$ & $-5.30(-6.65-3.96)$ & $<0.001$ & $<0.001$ \\
\hline \multicolumn{6}{|l|}{ Control } \\
\hline Current smoker, \% (n/N) & $6.8(14 / 207)$ & $9.0(22 / 244)$ & $1.869(0.759-4.598)$ & 0.173 & \\
\hline Diabetes, \% (n/N) & $18.8(39 / 207)$ & $23.4(57 / 244)$ & $1.371(0.843-2.231)$ & 0.204 & \\
\hline Newly diagnosed hypertension, \% (n/N) & $14.0(29 / 207)$ & $19.7(48 / 244)$ & $1.565(0.931-2.631)$ & 0.091 & \\
\hline History of CVD, \% (n/N) & $41.5(86 / 207)$ & $37.7(92 / 244)$ & $0.767(0.509-1.156)$ & 0.205 & \\
\hline $\mathrm{SBP}$, mean $\pm \mathrm{SD}$ & $142.28 \pm 16.58$ & $141.49 \pm 14.21$ & $-0.52(-3.36-2.32)$ & 0.721 & \\
\hline $\mathrm{DBP}$, mean $\pm \mathrm{SD}$ & $89.83 \pm 8.89$ & $89.85 \pm 9.29$ & $0.07(-1.63-1.77)$ & 0.932 & \\
\hline
\end{tabular}

CVD cardiovascular disease; SBP systolic blood pressure; $D B P$ diastolic blood pressure; $S D$ standard deviation; $O R$ odds ratio; $C l$ confidence interval

${ }^{a}$ Adjusted for age, gender, and clinic.

${ }^{b}$ Interaction for intervention and control clinics adjusted for age, gender, and clinic.

Among the intervention group, the proportion of hypertensive patients with blood pressure in normal range (primary outcome) significantly improved from baseline to follow-up (OR 3.556, $95 \%$ CI 2.219, 5.696); there was no significant change in the control group (OR 0.644, $95 \%$ CI 0.370, 1.121) (Table 4; Fig. 1). The improvement in the intervention groups was statistically significantly larger than the control ( $\mathrm{p}$ for interaction $<$ 0.001) (Table 4). This was also true for blood pressure control among patients with low WHO/ISH CVD risk scores but not for those with high CVD risk scores (Table 4; Fig. 1).

Of the process indicators, five significantly improved among the intervention group compared with control group. These were: the ability to calculate WHO/ISH CVD risk score, triple therapy prescribing for high risk patients, statin prescribing for high risk patients, recording of smoking status, and regular measurement of blood pressure (Table 4; Fig. 1). The remaining process indicators (total cholesterol testing, fasting glucose testing, prescription of blood pressure lowering medication, aspirin prescribing for high-risk patients), were not significantly different between intervention and control groups. At baseline, none of the records contained a documented $\mathrm{WHO} / \mathrm{ISH}$ risk score. This increased to $79.7 \%$ (295 of 370 records) at follow-up in the intervention group, in contrast to only $0.4 \%$ ( 1 of 242 records) in the control group. The proportion of patients with high CVD risk decreased significantly in intervention clinics, but was not significantly different than the change seen in control clinics (Table 4; Fig. 1).

\section{Discussion}

Baseline and follow-up collection and analysis of data from primary health care centres in Tajikistan demonstrated that it is feasible to collect quantitative data required for studies of effectiveness from routine clinical data. Furthermore, we were able to determine the baseline performance of primary health care services with respect to essential interventions for the management of hypertension and prevention of CVD, and their change over time. The intervention significantly improved blood pressure control, assessment of cardiovascular risk factors including blood pressure measurement and smoking status, and prescription of statins and triple therapy. It also significantly increased the proportion of newly diagnosed hypertension patients. It did not improve the testing rates of total cholesterol, fasting glucose tests, prescription of aspirin or blood pressure lowering drugs.

Measurement of biochemical risk factors is a basic part in the standard of care in the detection and management of NCDs yet the measurement of total cholesterol and fasting glucose were profoundly suboptimal. $[10,11]$ This is likely because of low availability and accessibility of these tests in primary health care, including having to pay out-of-pocket. Despite this, in resource limited settings it is still possible to CVD risk stratify individuals without access to laboratory testing and this was seen in the intervention group but not the control group.[9, 12, 13] Documentation of WHO/ISH CVD risk score improved from 0 to $79.7 \%$ in the intervention group illustrating a drastic uptake of CVD risk assessment independent of an increase in cholesterol testing. This is 
Table 4 Process and outcome indicators at baseline and 1-year follow-up

\begin{tabular}{|c|c|c|c|c|c|}
\hline \multirow[t]{2}{*}{ Process and outcome indicators } & \multirow{2}{*}{$\begin{array}{l}\text { Baseline } \\
\%(n / N)\end{array}$} & \multirow{2}{*}{$\begin{array}{l}\text { Follow-up } \\
\%(n / N)\end{array}$} & \multicolumn{2}{|c|}{ Baseline vs. Follow-up Difference ${ }^{a}$} & \multirow{2}{*}{$\begin{array}{l}\text { Interaction }^{\mathbf{b}} \\
P \text {-value }\end{array}$} \\
\hline & & & OR $(95 \% \mathrm{Cl})$ & $P$-value & \\
\hline \multicolumn{6}{|l|}{ Intervention } \\
\hline \multicolumn{6}{|l|}{ Process indicators } \\
\hline Smoking status recorded & $61.5(160 / 260)$ & $90.4(338 / 374)$ & 14.397 (7.987-25.886) & $<0.001$ & $<0.001$ \\
\hline BP measured regularly & $25.0(65 / 260)$ & $71.4(267 / 374)$ & $10.013(6.642-15.094)$ & $<0.001$ & $<0.001$ \\
\hline Fasting glucose tested & $13.1(34 / 260)$ & $16.3(61 / 374)$ & $1.393(0.860-2.255)$ & 0.178 & 0.800 \\
\hline Total cholesterol tested & $8.1(21 / 260)$ & $12.3(46 / 374)$ & $1.781(1.002-3.165)$ & 0.049 & 0.423 \\
\hline WHO/ISH risk score documented & $0.0(0 / 259)$ & $79.7(295 / 370)$ & - & - & - \\
\hline WHO/ISH risk score calculatable & $96.1(249 / 259)$ & $99.7(369 / 370)$ & $13.805(1.692-112.649)$ & 0.014 & 0.004 \\
\hline High-risk patients & $26.5(69 / 260)$ & $15.0(56 / 374)$ & $0.491(0.311-0.775)$ & 0.002 & 0.216 \\
\hline BP lowering drug prescribed & $96.5(251 / 260)$ & $97.1(363 / 374)$ & $1.568(0.596-4.124)$ & 0.362 & 0.910 \\
\hline Statin prescribed for high-risk patients & $23.2(16 / 69)$ & $78.6(44 / 56)$ & $12.640(4.365-36.599)$ & $<0.001$ & 0.001 \\
\hline Aspirin prescribed for high-risk patients & $85.5(59 / 69)$ & $87.5(49 / 56)$ & $0.368(0.091-1.493)$ & 0.162 & 0.213 \\
\hline Triple therapy prescribed for high-risk patients & $23.2(16 / 69)$ & $76.8(43 / 56)$ & 11.699 (3.947-34.679) & $<0.001$ & 0.001 \\
\hline \multicolumn{6}{|l|}{ Outcome indicators } \\
\hline $\mathrm{BP}$ at normal range & $14.8(34 / 229)$ & $32.5(118 / 363)$ & $3.556(2.219-5.696)$ & $<0.001$ & $<0.001$ \\
\hline High-risk patients with BP at normal range & $15.8(9 / 57)$ & $20.4(11 / 54)$ & $1.717(0.572-5.158)$ & 0.335 & 0.133 \\
\hline Low-risk patients with BP at normal range & $14.5(25 / 172)$ & $34.6(107 / 309)$ & $4.235(2.438-7.358)$ & $<0.001$ & 0.002 \\
\hline \multicolumn{6}{|l|}{ Control } \\
\hline \multicolumn{6}{|l|}{ Process indicators } \\
\hline Smoking status recorded & $73.4(152 / 207)$ & $68.0(166 / 244)$ & $0.395(0.210-0.743)$ & 0.004 & \\
\hline BP measured regularly & $62.3(129 / 207)$ & $68.9(168 / 244)$ & $1.319(0.849-2.049)$ & 0.218 & \\
\hline Fasting glucose tested & $22.7(47 / 207)$ & $30.7(75 / 244)$ & $1.554(0.959-2.519)$ & 0.074 & \\
\hline Total cholesterol tested & $12.1(25 / 207)$ & $15.2(37 / 244)$ & $1.257(0.681-2.321)$ & 0.464 & \\
\hline WHO/ISH risk score documented & $0.0(0 / 203)$ & $0.4(1 / 242)$ & - & - & \\
\hline WHO/ISH risk score calculatable & $99.5(202 / 203)$ & $98.3(238 / 242)$ & $0.185(0.015-2.328)$ & 0.192 & \\
\hline High-risk patients & $46.9(97 / 207)$ & $41.8(102 / 244)$ & $0.720(0.479-1.082)$ & 0.114 & \\
\hline BP lowering drug prescribed & $94.7(196 / 207)$ & $95.9(234 / 244)$ & $1.458(0.586-3.628)$ & 0.418 & \\
\hline Statin prescribed for high-risk patients & $13.4(13 / 97)$ & $15.7(16 / 102)$ & $1.610(0.529-4.903)$ & 0.402 & \\
\hline Aspirin prescribed for high-risk patients & $89.7(87 / 97)$ & $92.2(94 / 102)$ & $1.437(0.421-4.903)$ & 0.562 & \\
\hline Triple therapy prescribed for high-risk patients & $13.4(13 / 97)$ & $15.7(16 / 102)$ & $1.610(0.529-4.903)$ & 0.402 & \\
\hline \multicolumn{6}{|l|}{ Outcome indicators } \\
\hline $\mathrm{BP}$ at normal range & $17.8(36 / 202)$ & $13.2(31 / 234)$ & $0.644(0.370-1.121)$ & 0.120 & \\
\hline High-risk patients with BP at normal range & $22.1(21 / 95)$ & $13.0(13 / 100)$ & $0.571(0.235-1.389)$ & 0.216 & \\
\hline Low-risk patients with BP at normal range & $14.2(15 / 107)$ & $13.4(18 / 134)$ & $0.877(0.399-1.926)$ & 0.744 & \\
\hline
\end{tabular}

$B P$ blood pressure, $W H O / I S H$ risk score World Health Organization/International Society of Hypertension cardiovascular risk score; OR odds ratio; Cl confidence interval.

${ }^{\text {a }}$ Adjusted for age, gender, and clinic

${ }^{\mathrm{b}}$ Interaction for intervention and control clinics adjusted for age, gender, and clinic

possible since there are cholesterol-independent WHO/ ISH CVD risk charts.

Furthermore, using the cholesterol-independent WHO/ISH risk charts, we were able to calculate WHO/ ISH risk scores for nearly $100 \%$ of patients in both intervention and control groups. This demonstrated an important finding - while biochemical risk factor assessment is underutilized, it is still possible to translate existing clinical data into meaningful total cardiovascular risk scores without additional need for testing using WHO/ISH CVD risk charts. This is was true for both intervention and control groups, implying that given the 


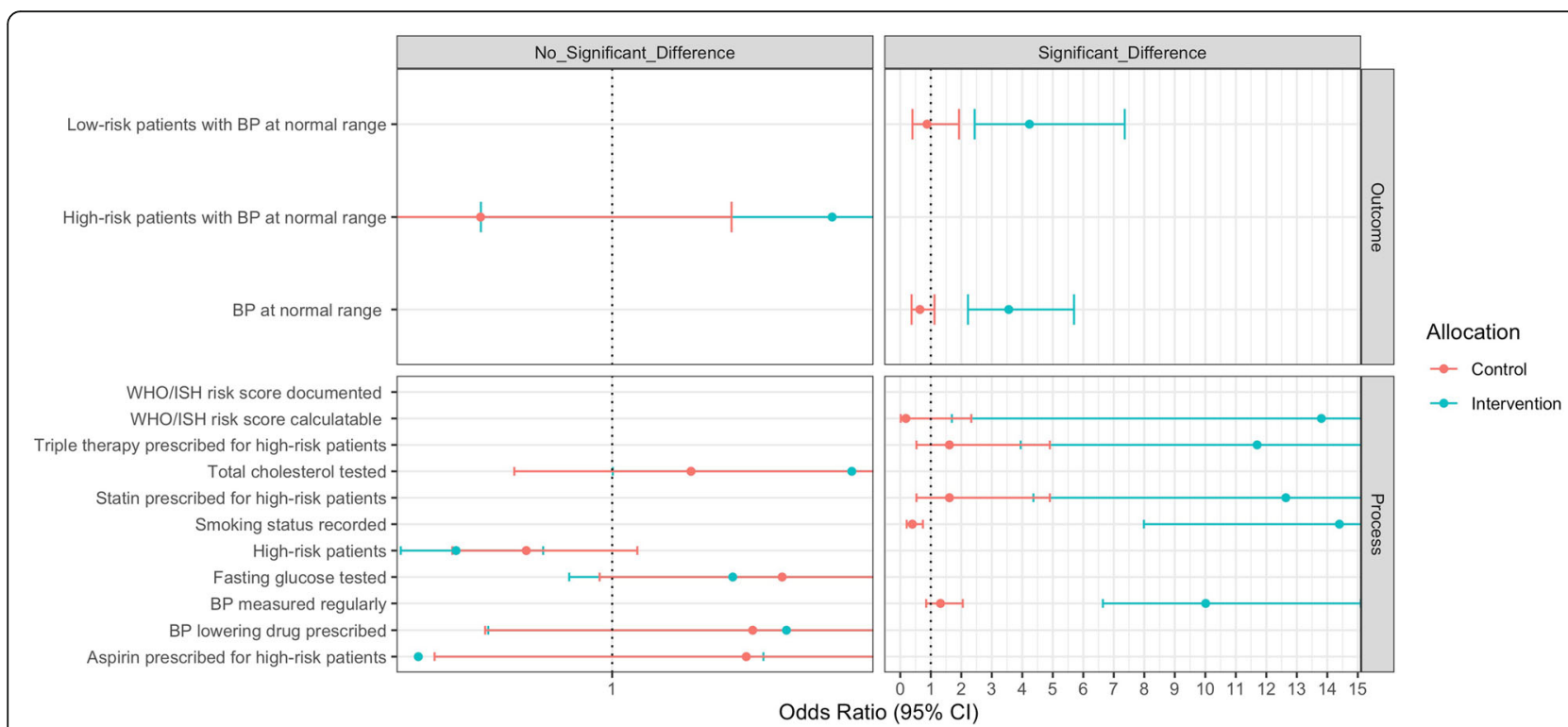

Fig. 1 Odds of achieving outcomes (odds ratio with $95 \% \mathrm{Cl}$ ) at 1-year follow-up compared to baseline for both intervention and control clinics

appropriate training and access to CVD risk charts, clinicians can quickly implement CVD risk scoring into their existing clinical workflow.

There was no significant difference in the rate of blood pressure lowering medication prescription between intervention and control groups, which was explained by the nearly perfect performance of this indicator at baseline. In the intervention and control groups, 96.5 and $94.7 \%$ of hypertensive patients, respectively, were prescribed blood pressure lowering medication. The observed effect on blood pressure reduction in intervention clinics may therefore be more strongly associated with the behavioural risk factor counselling and patient education materials that were part of the intervention.[14] The prescription of statins, however, significantly improved in the intervention group but not in the control group without changes in the rate of cholesterol testing. This is likely due to the fact that in the absence of cholesterol testing, statins can according to WHO PEN protocol be prescribed to those with high CVD risk scores including cases of secondary prevention.[5] Of course, it is unclear the degree to which these prescriptions are filled and the proportion who achieve medication adherence.

Results show a significant decline in blood pressure levels and proportion of patients with elevated blood pressure in intervention clinics compared with control clinics. However, no significant improvement was observed in prescription rates of blood pressure lowering medication, which was very high already at baseline. There may have been changes to the dosing of medication according to the HEARTS protocols, but unfortunately drug dosage information was not collected.
Increased medication adherence due to improved patient counselling may have also played a role. The decline in observed mean blood pressure in the intervention group can also be partly explained by the increased number of newly diagnosed patients with overall lower (but raised) blood pressure. The proportion of newly diagnosed hypertension patients increased significantly in the intervention clinics, which may be accounted for by increased screening. Forthcoming qualitative research will seek to explain these findings.

Previous research examining the quality of hypertension care in the Khatlon and Sogd oblasts of Tajikistan found persistent gaps in the detection, diagnosis, and outcomes of patients with hypertension.[15] In particular, it was estimated that only 5 to $10 \%$ of hypertensive patients actually receive a diagnosis. This is consistent with our findings in that the number of registered hypertensive patients in the Shahrinav oblast was far below the expected, which contributed to our lower than expected sample size. This is in part explained by insufficient supply of equipment (e.g. sphygmomanometers), high case load of primary health care workers, and lack of awareness among health administrators.[15] However, according to this evaluation study the detection rate of hypertension seems to have improved in intervention clinics in Shahrinav as both the number of patients recorded having hypertension as well as newly diagnosed patients increased.

The aspects of our intervention that may have contributed to the improvement in blood pressure control and several surrogate markers along the pathway of care, include a focus on known challenges to implementation and building on the previous work done nationally with 
WHO PEN. These lessons and experiences were used to adapt the HEARTS technical package to best meet known challenges to successful implementation. These include: using evidence-based, simplified, protocols designed for primary health care, conducting integrated training of doctors and nurses with task sharing and quality of care as part of the curriculum, the provision of decision support tools, an emphasis on grass roots quality improvement, and monitoring and feedback for quality improvement. [16] Without a comprehensive approach, however, taking into account organization processes, capacity to implement simplified clinical guidelines, the provision of essential equipment, medicines, and diagnostic tests further improvements may be limited. [17] Focus should be the continued prioritization of NCDs for public health intervention, optimizing clinical guidance for primary health care, engagement of key stakeholders, generating a local (i.e. Tajikistan) evidence base, and ensuring quality improvement while mainstreaming. [16]

Improvements in blood pressure control were also observed in the Republic of Moldova after the implementation of adapted WHO PEN guidelines and structured training for health workers. Compared to baseline, significant improvements were seen in intervention clinics at one year follow-up for patients with blood pressure at normal range, hypertensive patients with blood pressure at normal range, and patients with elevated blood pressure. Similar to our study, no significant change in aspirin prescriptions for CVD patients were observed. In contrast to our study, the prescriptions of statins for CVD patients did not change in intervention clinics and even deteriorated in control clinics.[18].

It was noted that the proportion of women increased in the follow-up (random) sample in both intervention and control clinics. We speculate that home visit programs that were not a part of our study that may have biased the clinic registers toward women over time as men are often not in the home during the day or are away for extended periods of time for work. This, and other important questions such as the adoption and perception of the intervention across the ten sites, the use of standard clinical algorithms and guidance, and perceived barriers will be examined in the qualitative arm of this study and is beyond the scope of this manuscript.

\section{Strength and Limitations}

This study is strengthened by its a priori publication of methods and its ability to be reproduced and scaled within the constraints of the existing Tajik health system. This work will be further strengthened by the planned qualitative research as part of a broader sequential explanatory mixed-methods design. There were four main limitations. First, the targeted sample size was not fully achieved as there were not enough registered hypertension patients to meet our target sample size. The study is therefore underpowered for some outcomes. Outcomes which did not show a statistically significant difference between intervention and control groups should therefore be judged in light of the possibility that if a difference did exist, this study may not detect it. Second, due to limited health record infrastructure, patients had to be sampled from the hypertension register so we were unable to assess patients without a formal diagnosis of hypertension. Third, for pragmatic reasons including the rugged and vast geography of Tajikistan, only one health region was included in the study and this may limit the generalizability of the findings although it is not known to be an outlier in terms of health system development or performance. Fourth, data collectors were unable to be blinded to allocation during the follow-up data collection due to the nature of the intervention.

\section{Conclusions}

It is feasible to use routine, paper-based, clinical records to evaluate essential CVD interventions in primary health care in Tajikistan. Pilot implementation of adapted WHO and HEARTS guidelines, including the systems for monitoring, demonstrated a significant impact on blood pressure control among hypertensive patients after 12 months, as well as several process indicators including CVD risk assessment. Continued monitoring and evaluation will be required as this intervention is scaled nationally.

\section{Abbreviations}

BMI: Body mass index; Cl: Confidence interval; CVD: Cardiovascular disease; ISH: International society of hypertension; NCD: Non-communicable disease; OR: Odds ratio; PEN: Package of essential NCD interventions for low-resource settings; WHO: World health organization

\section{Acknowledgements}

Authors wish to acknowledge the Ministry of Health and Social Protection of Population of Tajikistan, the Republican Clinical and Training Centre of Family Medicine, Service of State Supervision for medical activities and social protection of the population of the Republic of Tajikistan, the Institute for Postgraduate Education in Healthcare, Republican Health Information and Statistics Centre, and the district and rural managers and health care workers who participated in the study and its evaluation.

\section{Authors' contributions}

JF, TL, JF, DC, MS, DS, BJ planned the study protocol and JF, MS, DS, BJ organized its implementation. MS, DS and BJ organized the data collection and audited the quality of data. TL and $L I$ performed the quantitative data analyses, reviewed by DC. TL, JF and DC drafted the manuscript. KF, MA, MO contributed to data interpretation. All authors contributed to the interpretation of the results, critically reviewed and approved the final manuscript.

\section{Funding}

The WHO Regional Office for Europe have provided funding for the intervention and its evaluation, supported by grants from the US Centers for Disease Prevention and Control and the Government of the Russian 
Federation. The funders had no role in the methodological design or analysis of results.

\section{Availability of data and materials}

The datasets used and/or analysed during the current study are available from the corresponding author on reasonable request.

\section{Declarations}

Ethics approval and consent to participate

This project has been reviewed and granted ethical approval from the Republican Clinical Center for Family Medicine under the Ministry of Health and Social Protection of the Republic Tajikistan. As per the ethical approval review and the nature of this project, consent was not obtained from individual patients for their anonymized routine clinical data to be used.

\section{Consent for publication}

None declared.

\section{Competing interests}

Not applicable.

\section{Author details}

'University of British Columbia, Vancouver, Canada. ${ }^{2}$ Institute of Public Health and Clinical Nutrition, University of Eastern Finland, Kuopio, Finland. ${ }^{3}$ Finnish Institute for Health and Welfare, Helsinki, Finland. ${ }^{4}$ Joint municipal authority for North Karelia Health and Social Services, Joensuu, Finland. ${ }^{5}$ World Health Organization Regional Office for Europe, Copenhagen, Denmark. ${ }^{6}$ Service of State Supervision for Medical Activities and Social Protection of the Population of the Republic of Tajikistan, Dushanbe, Tajikistan. ${ }^{7}$ Republican Clinical and Training Centre of Family Medicine, Dushanbe, Tajikistan. ${ }^{8}$ Department of Epidemiology and Health Economics of the Faculty of Medicine, Tajik State University, Dushanbe, Tajikistan. ${ }^{9}$ Public Health, Faculty of Medicine, Social Hygiene and Health Organization, Tajik State University, Dushanbe, Tajikistan.

Received: 29 August 2020 Accepted: 6 May 2021

Published online: 18 May 2021

\section{References}

1. World Health Organization. Global Health Observatory - Total NCD Mortality by Country [Internet]. Geneva; 2018. Available from: http://apps.who.int/ gho/data/node.main.A857?lang=en.

2. World Health Organization. Risk of Premature Death from the Four Target NCDs. 2018.

3. Collins DRJ, Laatikainen T, Shoismatuloeva M, Mahmudzoha I, Rahimov Z, Sultonova D, et al. Evaluation and pilot implementation of essential interventions for the management of hypertension and prevention of cardiovascular diseases in primary health care in the Republic of Tajikistan [version 1; peer review: awaiting peer review]. F1000Research. 2019;8(1639).

4. WHO Regional Office for Europe. Review of the National Programme on the Development of Family Medicine 2011-2015 in Tajikistan. Copenhagen; 2016.

5. World Health Organization (WHO). Package of Essential Noncommunicable (PEN) disease interventions for primary health care in low-resource settings [Internet]. 2013. Available from: http://www.who.int/ncds/management/ pen_tools/en/.

6. Organization WH. Tackling, NCDs: "Best buys" and other recommended interventions for the prevention and control of noncommunicable diseases [Internet]. 2017. Available from: http://www.who.int/ncds/management/ best-buys/en/.

7. World Health Organizatin. HEARTS Technical Package. Geneva; 2018.

8. World Health Organization (WHO). Package of Essential Noncommunicable (PEN) Disease Interventions for Primary Health Care in Low-Resource Settings. 2010

9. Collins D, Lee J, Bobrovitz N, Koshiaris C, Ward A, Heneghan C. whoishRisk an $\mathrm{R}$ package to calculate $\mathrm{WHO} / \mathrm{ISH}$ cardiovascular risk scores for all epidemiological subregions of the world. F1000Research. 2016;5:2522.

10. Lall D, Engel N, Devadasan N, Horstman K, Criel B. Models of care for chronic conditions in low/middle-income countries: a 'best fit'framework synthesis. BMJ Glob Heal. 2018;3(6):e001077.
11. Wirtz V, Kaplan WA, Téllez YS-A, Ridaura RL. Affordable, quality, long-term care and pharmacotherapy of chronic diseases: a framework for low and middle income countries. Geneva, Switz World Heal Organ.; 2011.

12. World Health Organization. WHO/ISH Cardiovascular Risk Prediction Charts. Geneva; 2007

13. Collins DRJ, Jobanputra K, Frost T, Muhammed S, Ward A, Shafei AA, et al. Cardiovascular disease risk and prevention amongst Syrian refugees: mixed methods study of Medecins Sans Frontieres programme in Jordan. Confl Health. 2017;11:14

14. Baena CP, Olandoski M, Younge JO, Buitrago-Lopez A, Darweesh SKL, Campos $\mathrm{N}$, et al. Effects of lifestyle-related interventions on blood pressure in low and middle-income countries: systematic review and meta-analysis. J Hypertens. 2014;32(5):961-73.

15. Chukwuma A, Gong E, Latypova M, Fraser-Hurt N. Challenges and opportunities in the continuity of care for hypertension: a mixed-methods study embedded in a primary health care intervention in Tajikistan. BMC Health Serv Res. 2019;19(1):925.

16. Collins D, Laatikainen T, Farrington J. Implementing essential interventions for cardiovascular disease risk management in primary healthcare: lessons from Eastern Europe and Central Asia. BMJ Glob Heal. 2020;5(2).

17. World Health Organization (WHO). Global action plan for the prevention and control of NCDs 2013-2020. 2013.

18. Laatikainen T, Inglin L, Collins D, Ciobanu A, Curocichin G, Salaru V, et al. Implementing Package of Essential Non-communicable Disease Interventions in the Republic of Moldova-a feasibility study. Eur J Public Health. 2020;16:ckaa037. doi:https://doi.org/10.1093/eurpub/ckaa037.

\section{Publisher's Note}

Springer Nature remains neutral with regard to jurisdictional claims in published maps and institutional affiliations.

Ready to submit your research? Choose BMC and benefit from:

- fast, convenient online submission

- thorough peer review by experienced researchers in your field

- rapid publication on acceptance

- support for research data, including large and complex data types

- gold Open Access which fosters wider collaboration and increased citations

- maximum visibility for your research: over $100 \mathrm{M}$ website views per year

At BMC, research is always in progress.

Learn more biomedcentral.com/submissions 